\title{
BIOGRAFIAS INDÍGENAS: 0 EU E 0 OUTRO NA ESCRITA ANTROPOLÓGICA ${ }^{1}$
}

MESSIAS BASQUES ${ }^{2}$

PPGAS-UFRJ/MUSEU NACIONAL, BRASIL

RESUMO: entre as diversas abordagens que constituem o fazer antropológico contemporâneo, uma, em especial, praticamente não se desenvolveu na história da disciplina no Brasil: a biografia. Este ensaio apresenta um panorama da relação entre a etnologia indígena e o gênero biográfico, com foco nas noções de pessoa e indivíduo e em suas implicações para a escrita antropológica.

PALAVRAS-CHAVE: biografia, autobiografia, etnobiografia, antropologia, etnologia indígena

\begin{abstract}
: among the many approaches that constitute contemporary anthropological practices, one, in particular, has hardly developed in the history of the discipline in Brazil: biography. This essay presents an overview of the relationship between anthropology and the biographical genre, focusing on the notions of person and individual and their implications for anthropological writing. The essay focuses on the area of indigenous ethnology and, in particular, on anthropological studies of Amerindian peoples.
\end{abstract}

KEYWORDS: biography, autobiography, etnobiography, anthropology, indigenous ethnology

\footnotetext{
${ }^{1}$ Este texto foi originalmente redigido pelo autor para o projeto "Histórias ocultas: vidas indígenas no Brasil republicano", que concorreu ao Edital 13/2015 - "Memórias brasileiras: biografias"/CAPES, sob a coordenação-geral do prof. Dr. Carlos Fausto. O projeto não foi contemplado pelo Edital. A equipe proponente era composta pelos seguintes pesquisadores: Carlos Cesar Leal Xavier, Danielle Araujo Bueno dos Santos, Elena Monteiro Welper, Etelvino de Almeida, Hélio Cabral de Sá Neto, José Ninha Tavares Kanamari, Laércio Barbosa Victor, Luana Machado de Almeida, Luiz Antônio Lino da Silva Costa, Luiz Fabiano de Freitas Tavares, Mutua Mehinaku Kuikuro, Nelly Barbosa Duarte Dollis, Nian Pissolati Lopes, Paulo Leme Gonzalez Büll, Takumã Kuikuro, Thayná Ferraz da Cunha Pinheiro, Thiago Lopes da Costa Oliveira, Yamalui Mehinaku Kuikuru.

${ }^{2}$ Doutorando em Antropologia Social (Universidade Federal do Rio de Janeiro / Museu Nacional, Brasil). E-mail: messias.basques@gmail.com
} 


\section{Introdução}

$\mathrm{Na}$ história da antropologia, a etnografia se constituiu como o principal avatar da disciplina, embora não se possa dizer que haja consenso quanto à definição do que é a etnografia: um conjunto de técnicas de pesquisa; um modo de observação e interpretação aplicável a diversos objetos de estudo; um regime da escrita antropológica; uma relação de conhecimento que se baseia na experiência da alteridade (INGOLD, 2014). Tampouco se pode dizer que os cursos de Ciências Sociais ofereçam aos seus alunos uma formação acadêmica estritamente padronizada, cuja estrutura curricular tenha como resultado um profissional reconhecido como etnógrafo. Uma das contrapartidas dessa indefinição talvez seja a abertura da disciplina e de seus praticantes a uma vasta gama de objetos de estudo, de modos de investigação, de escrita e de produção de conhecimento. Entre as inúmeras abordagens que constituem o fazer antropológico contemporâneo, uma, em especial, praticamente não se desenvolveu na história da disciplina no Brasil: a biografia.

O compromisso da disciplina com o horizonte de comparação e com o diálogo entre diferentes campos de estudo parece colocado em xeque quando se trata de pensar os usos da biografia e da autobiografia como práticas antropológicas de mesmo estatuto que a etnografia. O que se costuma encontrar nas publicações da área é o uso sobretudo acessório das histórias de vida na escrita etnográfica (GONÇALVES et al., 2012; KOFES \& MANICA, 2015, p.16; OAKDALE \& COURSE, 2014, p.1; SÁEZ, 2006, p.189). O cenário brasileiro é bastante distinto, porém, daquele encontrado em países da América Latina, da Oceania e da América do Norte, onde "as histórias de vida têm sido um recurso essencial da pesquisa etnográfica e a autobiografia indígena virou, desde muito cedo, um gênero central da literatura escrita por índios" (SÁEZ, 2012, p.14-5). Alguns autores sugerem que as imagens sedimentadas na opinião pública, no âmbito acadêmico e na ordem jurídica brasileira obliteram e negligenciam as formas individuais do pensamento e da vida indígenas, como se o sujeito indígena não pudesse ser outra coisa que um suposto sujeito coletivo (SÁEZ, 2007, p.11; BASSO, 1989, p.551; BASSO, 2003), o que sem dúvida não ocorre quando consideramos o enorme sucesso editorial dos relatos autobiográficos no Ocidente.

Se a literatura etnológica brasileira se caracteriza pela incipiência de publicações desse gênero, o mesmo já não se pode dizer da própria literatura indígena, que passou por um recente florescimento, especialmente entre os povos da bacia do Rio Negro (SÁEZ, 2012 p.12), mas não apenas. Tais publicações também têm se convertido em importantes plataformas para a atuação e consolidação política de lideranças indígenas, bem como contribuem para uma maior visibilidade desses povos, de seu pensamento e de suas demandas no âmbito nacional e internacional (OAKDALE \& COURSE, 2014, p.8; BACIGALUPO, 2014; VIENNE, 2014; KOPENAWA \& ALBERT, 2015; RAONI \& DUTILLEUX, 2010). Como sugere Bruce Albert (1997, p.59), “a fórmula da 
autorrepresentação" [se tornou] "um instrumento político altamente efetivo", o que fica claro no caso exemplar da líder indígena guatemalteca Rigoberta Menchú, Prêmio Nobel da Paz, cuja autobiografia a projetou internacionalmente.

Nos Estados Unidos, o gênero biográfico com foco em personalidades indígenas difundiu-se muito antes do que no restante das Américas (BRUMBLE, 2008; KRUPAT, 1994), talvez em função da importância cultural do indivíduo no contexto protestante americano, da maneira pela qual se deu a invasão das terras indígenas e a concessão de reservas (DARNELL, 2001). Segundo Sáez (2006, p.180), "a primeira metade do século XX é o momento em que a autobiografia indígena é promovida pelos antropólogos, inspirados - embora não exclusivamente - pelas preocupações da escola de Cultura e Personalidade". Naquele momento, buscava-se nesses depoimentos não só informações etnológicas, mas principalmente "uma aproximação mais imediata à alma indígena e às relações entre indivíduo e padrão cultural" (:ibid.). Paul Radin foi um dos precursores desse tipo de abordagem. Em 1926, ele publicou a autobiografia de um indígena Winnebago e chamava atenção para o fato de que não se tratava de uma biografia heroica, na qual são celebradas as virtudes e a trajetória individual do biografado, mas antes uma forma de reflexão sobre uma vida que se construía na própria narração.

Trinta anos atrás, a antropóloga Ellen Basso (1989, p.551) indicava que o estudo de narrativas pessoais estava se tornando cada vez mais relevante, acompanhando assim uma guinada da disciplina dos estudos conduzidos sob o viés estruturalista para aqueles mais preocupados com a análise do discurso e da subjetividade. Os estruturalismos, tanto inglês quanto francês, hegemônicos entre os anos 1940 e 1970, inibiram o investimento na escala biográfica, ainda que Lévi-Strauss (1982) tenha, ele mesmo, escrito o prefácio à tradução francesa da biografia do chefe Hopi Don C. Talayesva, em que afirma que essa "joia da literatura etnográfica" torna possível aos etnólogos a realização de um sonho: olhar uma cultura desde o seu interior, ou melhor, a partir dos olhos de um nativo (ver também LÉVI-STRAUSS, 1943).

Stewart \& Strathern (2000) defendem que essas abordagens permitem à antropologia a consideração das relações entre pessoas, biografias e história, temas que poucas vezes aparecem com a mesma importância e espaço na literatura. As narrativas, sejam elas de ordem mitológica, histórica ou (auto)biográfica, expõem distintos modos de conceber e de construir a temporalidade, assim como podem auxiliar a compreensão da inscrição de eventos históricos em formas discursivas e imagéticas (BASSO, 1995; CARNEIRO DA CUNHA, 1992; FAUSTO, 2002; FAUSTO \& HECKENBERGER, 2007; FRANCHETTO, 1993; GALLOIS, 1994; HENDRICKS, 1993; KOHN, 2002; MACCLANCY, 2007; OAKDALE, 2005).

Nas sociedades indígenas, as narrativas orais são fundamentais para a construção dos regimes de historicidade (BANNER, 2009; HARTOG, 2002), como é sublinhado por Anne-Christine Taylor (2007, p.147) a partir de suas pesquisas com os Achuar no Equador, para os quais as formas autobiográficas são parte importante da construção de um 
"regime de história". O trabalho de Suzanne Oakdale (2005) entre os Kayabi do Baixo Xingu, por sua vez, revela que a abordagem biográfica pode nos oferecer uma compreensão não apenas da historicidade indígena, mas também da noção de pessoa que lhe é correspondente. A autobiografia transita, assim, entre os mais diversos discursos Kayabi, refletindo-se na fala política dos líderes, nos cantos bélicos e xamânicos.

Ana Bacigalupo (2014) sugere que as biografias xamânicas entre os Mapuche do Chile fornecem testemunhos que não se encerram nos sujeitos que os enunciam, uma vez que nos permitem conhecer diversos aspectos da cosmopolítica indígena, bem como entender a dialética entre continuidade e mudança. Biógrafos e biografados são vistos e reconhecidos como pessoas que vivem na dobra de uma relação que se dá entre as figuras da individualidade e da coletividade a qual pertencem. A intersecção entre um percurso singular e uma forma coletiva expressase não apenas na forma de narrativas, mas também na forma de imagens rituais (FAUSTO \& PENONI, 2014; FAUSTO \& SEVERI, 2014; GUERREIRO JR., 2011). Assim, por exemplo, no caso do Alto Xingu, a efígie do Quarup é chamada pelos Kuikuro de X-hutoho, onde $X$ é o nome do chefe representado pelo artefato. A efígie é a figuração de uma pessoa com uma biografia particular, mas é, ao mesmo tempo, um índice da condição geral da chefia, de uma pessoa magnificada, que contém não apenas uma história pessoal, mas uma história coletiva (FAUSTO, 2012). Ao passo que na festa do Javari, embora a efígie possua atributos da identidade de alguém, a sua função mais evidente é a figuração de outrem: os primosadversários.

Mais do que um sintoma daquilo que Marshall Sahlins (2006, p.142) chamou de "moderna sujeitologia", esses estudos demonstram que as narrativas biográficas possuem um "lugar de direito" entre os povos indígenas nas Terras Baixas Sul-Americanas, tanto nos domínios da reflexão onírica, artística ou divinatória, quanto no plano da atuação e expressão de lideranças indígenas. Em uma coletânea publicada recentemente, Oakdale $\&$ Course (2014) sugerem que podemos pensar a constituição de biografias (e biografados) indígenas nas Terras Baixas a partir de composições de caráter "centrípeto" ou "centrifugo"; i.e., respectivamente, a partir de biografias (e biografados) que se concebem sob a lógica da incorporação de elementos externos, ou, alternativamente, a partir da constituição de uma pessoa que se destaca, magnificando-se, tornando-se reconhecida e reconhecível entre os seus e os outros. Fabre et al. (2010, p.20), na edição da revista L'Homme dedicada ao tema, propõem que as biografias podem servir como uma experiência que interroga os seus próprios fundamentos e explora, assim, a diversidade cultural e histórica dos modos de conceber o que é uma vida. Em outras palavras, ao explorar biografias indígenas é preciso estar atento às diferenças nos modos de conceber o sujeito, a relação entre a ação e a sua fonte, bem como a unidade espaço-temporal representada por uma vida. 


\section{Os dilemas da biografia indígena}

Desde o artigo de Herbert Baldus (1937) sobre o bororo Tiago Aipobureu, poucas foram as iniciativas de elaboração de biografias indígenas no Brasil. Mesmo o livro História dos Índios no Brasil (1992), que se tornou uma obra de referência, não faz qualquer menção à palavra biografia em suas quase 700 páginas; exceto por uma menção à biografia do Marechal Rondon. É digno de nota que entre os anos de 1904 e 1908, a Revista do Instituto Histórico e Geográfico do Rio Grande do Norte tenha criado uma seção intitulada "Índios Célebres", em torno da qual se desenrolou a polêmica acerca da naturalidade do indígena Antônio Felipe Camarão (1600/1601-1648) e onde também seria louvada a sua contribuição no conflito travado contra os holandeses na capitania de Pernambuco. Nos anos 2000, o historiador Ronaldo Vainfas coordenou a edição do Dicionário do Brasil Colonial (1500-1808), no qual foram incluídos verbetes biográficos acerca de indígenas como Antônio Paraupaba, Araribóia, Tibiriçá, Zorobabé, entre outros. O que não ocorreu no Dicionário do Brasil Imperial (1822-1889), obra que não contém nenhum verbete biográfico dedicado a personagens indígenas.

Uma das dificuldades para o desenvolvimento de biografias indígenas é a associação, quase espontânea, entre o gênero biográfico e a noção ocidental de indivíduo (LOCKE, 1995; DUMONT, 1977). Isso coloca desafios particulares à construção de biografias indígenas, em particular quando se pretende incorporar as concepções indígenas no próprio produto final. Trata-se menos de trazer à luz histórias ocultas e desconhecidas pelo grande público ou pela própria antropologia, mas de fazê-lo de modo a não reduzir o mundo e as concepções indígenas ao peso de um modo predeterminado de narrar a história de indivíduos.

Nas sociedades indígenas das Terras Baixas da América do Sul, a pessoa é constituída por múltiplas relações com outros seres humanos e não-humanos (animais, plantas, espíritos...), sem que se postule um núcleo fixo e estável, que responda pela identidade pessoal através do tempo e do espaço (FAUSTO, 2008; VILACA, 2002; SEEGER et al., 1979; TAYLOR, 1996; TURNER, 1995). A concepção hegemônica de pessoa no Ocidente, a qual está na base do próprio gênero (auto)biográfico, é completamente diversa e depende, ainda, de inúmeros dispositivos de construção de uma subjetividade que pode ser narrada. Este não é o caso das sociedades indígenas, as quais não apenas esposam teorias diversas da pessoa, como possuem dispositivos diversos de subjetivação e de narração.

As formas narrativas indígenas que se aproximam ao que reconhecemos como o gênero autobiográfico - ou seja, aquele no qual alguém narra a sua singularidade para outras pessoas - não são, contudo, tão incomuns entre os ameríndios (SÁEZ, 2006, p.185-188). É preciso levá-las em consideração, desde que partamos do reconhecimento de certas especificidades. Em primeiro lugar, elas muitas vezes seguem fórmulas poéticas ou musicais padronizadas, o que as remetem a convenções discursivas que inibem a manifestação da individualidade 
(DÉLÉAGE, 2007). Em segundo lugar, elas se inserem num regime complexo de citações, no qual o narrador se confunde com outras vozes, criando o efeito de uma pessoa múltipla - uma pessoa que cita outros citando outros, num espiral discursivo que dificulta a identificação de quem, de fato, está narrando, e quem, de fato, é o agente dos atos narrados (e.g. VIVEIROS DE CASTRO, 1992, p.227-234; OAKDALE, 2008; CESARINO, 2011 , p.205-228, 2014). Em terceiro lugar, elas não seguem uma narrativa linear de eventos envolvendo o narrador, mas incorporam temas míticos, sonhos e eventos que ocorreram a terceiros (como os parentes, por exemplo), assim tecendo uma sobreposição de registros que embaralha o regime temporal e ultrapassa a experiência fenomenal (e.g. HENDRICKS, 1990; GRAHAM, 1995; HIGH, 2014). Por fim, o narrador nem sempre busca estabelecer um vínculo com a história narrada, ou transmitir eventos que ocorreram em outros tempos e lugares, mas antes uma relação com os ouvintes no presente (GOW, 2001, p.78-92, 2014; OAKDALE, 2005, p.68-70).

Se estas particularidades marcam o gênero das autobiografias indígenas de forma geral, as biografias de pessoas já falecidas apresentam ainda outros desafios. Metodologicamente, dependemos de histórias orais, reconstruções de trajetórias de indivíduos que nossos interlocutores conheceram pessoalmente, mas que os pesquisadores não conheceram. Lidamos, assim, com a complexidade adicional das vicissitudes dos regimes de memória indígenas, e da narração de histórias que podem envolver os nossos interlocutores, mas que têm por objeto a pessoa já falecida. Nestas narrativas, a imbricação de mito e história, cosmologia e biografia, tende a ser ainda mais acentuada.

É fundamental considerar as particularidades das biografias indígenas não como empecilhos, mas como aspectos que nos permitem conceber, enquanto antropólogos, contribuições originais ao gênero biográfico. Se levadas em conta, elas oferecem possibilidades ímpares para o desenvolvimento de verdadeiras biografias indígenas (e não "biografias de índios"). O desenvolvimento de abordagens biográficas, ancoradas em fontes documentais e em uma relação dialógica com os povos indígenas, representa um campo promissor e praticamente inexplorado pela antropologia brasileira. Nos últimos anos, foram publicadas duas coletâneas com pesquisas antropológicas realizadas com diferentes abordagens biográficas (KOFES \& MANICA 2015; GONÇALVES et al. 2012), mas nenhuma cujo objetivo seja a descrição de personagens indígenas. 


\section{Referências Bibliográficas}

ALBERT, B. Etnographic situation and Ethnic movements: notes on post-Malinowskian fieldwork. Critique of Anthropology 17(1), p.53-65, 1997.

BACIGALUPO, A. The potency of indigenous 'bibles' and biographies: Mapuche shamanic literacy and historical consciousness. American Ethnologist 41(4), p.648-663, 2014.

BALDUS, H. O professor Tiago Marques e o caçador Aipobureu: a reação de um índio bororo à influência da nossa civilização. In: Ensaios de Etnologia Brasileira. São Paulo: Editora Nacional. p.92-107, 1979 [1937].

BANNER, L. W. Biography as History. The American Historical Review, 114(3), p.579586, 2009.

BASSO, E. Translating "Self-Cultivation.” In: MARANHÃO, T.; STRECK, B. (Eds.) Translation and Ethnography: The Anthropological Challenge of Intercultural Understanding. Tucson: University of Arizona Press, p.85-101, 2003.

BASSO, E. B. Kalapalo Biography: Psychology and Language in a South American Oral History. American Anthropologist 91(3), p.551-569, 1989.

BASSO, E. B. The Last Canibals: A South American Oral History. Austin: University of Texas Press, 1995.

BRUMBLE, H. D. American Indian Autobiography. Berkeley: University of California Press, 2008 [1988].

CARNEIRO DA CUNHA, M. (Org.). História dos índios no Brasil. São Paulo: Companhia das Letras, Secretaria Municipal de Cultura, FAPESP, 1992.

CESARINO, P. N. Oniska: poética do xamanismo na Amazônia. São Paulo: Perspectiva/FAPESP, 2011.

CESARINO, P. N. Multiple biographies: shamanism and personhood among the Marubo of western Amazonia. In: COURSE, M.; OAKDALE, S. (Orgs.). Fluent Selves: Autobiography, Person and History in Lowland South America. Omaha: University of Nebraska Press, p.121-143, 2014.

DARNELL, R. Invisible Genealogies: a History of Americanist Anthropology. Omaha: University of Nebraska Press, 2001.

DÉLÉAGE, P. A Yaminahua Autobiographical Song: Caqui Caqui. Tipití: Journal of the Society for the Anthropology of Lowland South America, v. 5, n. 1, p.79-25, 2007. DUMONT, L. Homo aequalis: genèse et épanouissement de l'idéologie économique. Paris: Gallimard, 1977. 
FABRE, D. et al. Jeu et enjeu ethnographiques de la biographie. L'Homme 195/196, p.720, 2010.

FAUSTO, C. \& HECKENBERGER, M. Introduction: Indigenous History and the History of the Indians. In: FAUSTO, C.; HECKENBERGER, M. (Eds.). Time and Memory in Indigenous Amazonia: Anthropological Perspectives. Gainesville, FL: University of Florida Press, p.1-43, 2007.

FAUSTO, C. \& PENONI, I. The effigy, the cousin and the dead: An essay on the Javari ritual (Upper Xingu, Brazil). Mimeo, 2014.

FAUSTO, C. Donos demais: Maestria e domínio na Amazônia. Mana, 14(2), p.329-366, 2008.

FAUSTO, C. Faire Ie mythe: Histoire, recit et transformation en Amazonie. Journal de la Société des Américanistes, v. 88, p.69-90. 2002.

FAUSTO, C. Warfare and Shamanism in Amazonia. New York: Cambridge University Press, 2012.

FRANCHETTO, B. Autobiographies of a memorable mand and other memorable persons (Southern Amazonia, Brazil). In: OAKDALE, S. \& COURSE, M. Fluent Selves: autobiography, person, and history in Lowland South America. Lincoln: Nebraska University Press, p.271-309, 2014.

FRANCHETTO, B. "A celebração da história nos discursos cerimoniais kuikuro (Alto Xingu)". In: M. Carneiro da Cunha \& E. viveiros de Castro (orgs.), Amazônia: etnologia e história indígena. São Paulo: Núcleo de História Indígena e do Indigenismo da USP/ Fapesp. p. 95-116, 1993.

GALLOIS, D. Mairi revisitada: a reintegração da Fortaleza de Macapá na tradição oral dos Waiãpi. Sao Paulo: NHII-USP/FAPESP, 1994.

GONÇALVES, M.A.; MARQUES, R. \& CARDOSO, V.Z. (Orgs.). Etnobiografia: subjetivação e etnografia. Rio de Janeiro: 7 Letras, 2012.

GOW, P. "This happened to me": exemplary personal experience narratives among the Piro (Yine) people of Peruvian Amazonia'. In: COURSE, M.; OAKDALE, S. (Orgs.). Fluent Selves: Autobiography, Person and History in Lowland South America. Omaha: University of Nebraska Press, p. 69-92, 2014.

GOW, P. An Amazonian Mith and Its History. Oxford Studies in Social and Cultural Anthropology. Oxford: Oxford University Press, 2001.

GRAHAM, L. Performing Dreams: Discourses of Immortality among the Xavante of Central Brazil. Austin: University of Texas Press, 1995.

GUERREIRO JR., A. Esteio de gente: reflexões sobre assimetria e parentesco a partir de depoimentos de chefes kalapalo. Revista de Antropologia Social dos Alunos do PPGASUFSCar, v. 3, n. 1, p. 95-126, 2011. 
HARTOG, F. Regimes of Historicity. Presentism and experiences of time. Columbia: Columbia University Press, 2002.

HENDRICKS, J. Manipulating time in an Amazonian society: genre and event among the Shuar. In: BASSO, E. (Ed). Native Latin American Cultures Through Their Discourse. Bloomington: Special Publications of the Folklore Institute, p.11-28, 1990.

HENDRICKS, J. To Drink of Death: the narrative of a Shuar Warrior. Tucson: University of Arizona Press, 1993.

HIGH, C. Like the Ancient Ones: The Intercultural Dynamics of Personal Biography in Amazonian Ecuador. In: OAKDALE, S. \& COURSE, M. (Eds). Fluent Selves: Autobiography and personhood in Lowland South America. Lincoln: University of Nebraska Press, p.35-68, 2014.

INGOLD, T. "That's enough about ethnography!”. Hau: Journal of Ethnographic Theory 4 (1), p.383-395, 2014.

KOFES, S. \& MANICA, D. (Orgs.). Vida e Grafias: narrativas antropológicas, entre biografia e etnografia. Rio de Janeiro: Lamparina \& FAPERJ, 2015.

KOHN, E. Infidels, Virgins, and the Black-Robed Priest: A Backwoods History of Ecuador's Montaña Region. Ethnohistory, v. 49, n. 1, p.545-582, 2002.

KOPENAWA, Davi \& ALBERT, Bruce. A queda do céu: Palavras de um xamã yanomami. Tradução de Beatriz Perrone-Moisés; prefácio de Eduardo Viveiros de Castro. São Paulo: Companhia das Letras, 2015.

KRUPAT, A. Native American Autobiography. Madison: University of Wisconsin Press, 1994.

LÉVI-STRAUSS, C. Review of L. Simmon's Sun Chief, The Autobiography of a Hopi Indian. Social Research, v. 10, n. 4, p.515- 517, 1943.

LÉVI-STRAUSS, Claude. "Préface”. Don Talayvesa, Soleil Hopi, Paris: Plon, 1982.

LOCKE, J. An essay concerning human understanding. Oxford: Clarendon Press, [1691] 1995.

MACCLANCY, J. Nakomaha: a counter-colonial life and its contexts. Anthropological approaches to biography. Oceania, v. 77, p.191-214, 2007.

OAKDALE, S. \& COURSE, M. Fluent Selves: autobiography, person, and history in Lowland South America. Lincoln: Nebraska University Press, 2014.

OAKDALE, S. I Foresee My Life: The Ritual Performance of Autobiography in an Amazonian Community. Lincoln: University of Nebraska Press, 2005. 
Espaço Ameríndio

OAKDALE, S. Creating a continuity between self and other: first-person narration in an Amazonia ritual context. Ethos, v.30, n.1-2, p.158-175, 2008.

PETRULlO, V. Primitive peoples of Mato Grosso. The Museum Journal. Philadelphia, v. 23, n. 2, p.91-78, 1932.

RADIN, P. Crashing Thunder: The Autobiography of an American Indian. New York: D. Appleton \& Co, 1926.

RAONI \& DUTILLEUX, Jean Pierre. Memoirs of an Indian Chief. Paris: Rocher, 2010. SÁEZ, Oscar C. "Autobiografia e sujeito histórico indígena”. Novos Estudos CEBRAP, 76, p.179-195, 2006.

SÁEZ, O. C. “Autobiografia e liderança indígena no Brasil”. Tellus 7(12), p.11-32, 2007.

SÁEZ, O. C. História indígena, autoria e sexo: a obra inédita de Gabriel Gentil. Tellus, v. 12, n. 22, p.11-26, 2012.

SAHLINS, M. História e cultura: apologias a Tucídides. Rio de Janeiro, Jorge Zahar, 2006.

SEEGER, A.; DA MATTA, R.; \& VIVEIROS DE CASTRO, E. A construção da pessoa nas sociedades indígenas brasileiras. Boletim do Museu Nacional, v. 32, p. 2-19, 1979.

SEVERI, C. \& FAUSTO, C. (Eds.). L’image rituelle. Paris: L'Hernes, 2014.

STEWART, P.J. \& A. STRATHERN. “Introduction: narratives speak". In: P.J. Stewart \& A. Strathern (eds). Identity work. Constructing Pacific lives. Pittsburgh: University of Pittsburgh Press, p.1-26, 2000.

TALAYESVA, D. Sun Chief: The Autobiography of a Hopi Indian. Second Edition. Yale: Yale University Press, 2013.

TAYLOR, A-C. Sick of history: Contrasting regimes of historicity in the Upper Amazon. In: FAUSTO, C. \& HECKENBERGER, M. (Eds.). Time and memory in indigenous Amazonia: Anthropological perspectives. Gainesville: University Press of Florida, p. p.133-68, 2007.

TAYLOR, A-C. The soul's body and its states. Journal of The Royal Anthropological Institute, v. 2, p- 201-215, 1996.

TURNER, T. Social body and embodied subject: bodylines, subjectivity and sociality among the Kayapó. Cultural Antropology, v. 10, p.143-170, 1995.

VAINFAS, R. (Org). Dicionário do Brasil Colonial (1500-1808). Rio de Janeiro: Objetiva, 2000.

VAINFAS, R. (Coord.). Dicionário do Brasil Imperial (1822-1889). Rio de Janeiro: Objetiva, 2002. 
Espaço Ameríndio

VIENNE, E. de. "Comment on kopenawa, Davi and Bruce Albert. 2013. The falling sky: Words of a Yanomami shaman". Hau: Journal of Ethnographic Theory 4 (2), p.311$317,2014$.

VILAÇA, A. Making kin out of others in Amazonia. The Journal of the Royal Anthropological Institute, v. 8, n. 2, p.347-365, 2002.

VIVEIROS DE CASTRO, E. From the enemy's point of view: humanity and divinity in an Amazonian society. Chicago: University of Chicago Press, 1992.

Recebido em: 19/06/2019* Aprovado em: 14/08/2019* Publicado em: 30/12/2019 